\title{
Bootstrap prediction mean squared errors of unobserved states based on the Kalman filter with estimated parameters
}

\author{
Alejandro Rodríguez ${ }^{\mathrm{a}, *}$, Esther Ruiz ${ }^{\mathrm{b}}$ \\ ${ }^{a}$ Departamento de Estadística, Universidad de Concepción, Avda. Esteban Iturra s/n, Barrio Universitario, 403200, Concepción, Chile \\ ${ }^{\mathrm{b}}$ Departamento de Estadística and Instituto Flores de Lemus, Universidad Carlos III de Madrid, Calle Madrid 126, 28903, Getafe (Madrid), Spain
}

\section{Keywords:}

NAIRU

Output gap

Parameter uncertainty

Prediction intervals

State space models

\begin{abstract}
A B S T R A C T
In the context of linear state space models with known parameters, the Kalman filter (KF) generates best linear unbiased predictions of the underlying states together with their corresponding Prediction Mean Square Errors (PMSE). However, in practice, when the filter is run with the parameters substituted by consistent estimates, the corresponding PMSE do not take into account the parameter uncertainty. Consequently, they underestimate their true counterparts. In this paper, we propose two new bootstrap procedures to obtain PMSE of the unobserved states designed to incorporate this latter uncertainty. We show that the new bootstrap procedures have better finite sample properties than bootstrap alternatives and than procedures based on the asymptotic approximation of the parameter distribution. The proposed procedures are implemented for estimating the PMSE of several key unobservable US macroeconomic variables as the output gap, the Non-accelerating Inflation Rate of Unemployment (NAIRU), the long-run investment rate and the core inflation. We show that taking into account the parameter uncertainty may change their prediction intervals and, consequently, the conclusions about the utility of the NAIRU as a macroeconomic indicator for expansions and recessions.
\end{abstract}

\section{Introduction}

State space models are very popular for describing the dynamic evolution of a large range of economic and financial time series in which there are unobserved variables of interest; see, for example, Fernández-Villaverde et al. (2007) who propose representing the equilibrium of an economic model using a state-space representation, Orphanides and van Norden (2002), Doménech and Gómez (2006) and Proietti et al. (2007) for estimating the unobserved output gap in several economies and Stock and Watson (2007) for a trend-cycle model with stochastic volatility fitted to US inflation, just to cite several recent empirical applications.

One of the main attractiveness of state space models is that they allow the implementation of the Kalman filter and smoothing algorithms which deliver estimates of the underlying states which, in the context of linear state space models with known parameters, are best linear unbiased. The filters also deliver the corresponding prediction mean squared errors (PMSE) which measure the uncertainty associated with the estimated states. However, in practice, the filter is run with some parameters substituted by consistent estimates. In this case, the Kalman filter PMSE do not take into account the additional uncertainty due to the parameter estimation. As a result, they underestimate the true PMSE and, consequently, the uncertainty associated with the estimates of the underlying states; see among others, Ansley and Kohn (1986), Hamilton (1986), Durbin and Koopman (2000) and Quenneville and Singh (2000).

\footnotetext{
* Corresponding author. Tel.: +56 412203336 ; fax: +56 412251529.

E-mail addresses: aleferodriguez@udec.cl, alejandrofederico.rodriguez@gmail.com (A. Rodríguez), ortega@est-econ.uc3m.es (E. Ruiz).
} 
There are several procedures available in the literature to incorporate the parameter estimation uncertainty into the Kalman filter PMSE. First, fully Bayesian methods generate distributions of the underlying states which in a natural way incorporate the parameter uncertainty; see, for example, Carter and Kohn (1994) and Durbin and Koopman (2002). However, these procedures can be computationally complicated and time consuming in relatively large models; see Harvey (2000) and Quenneville and Singh (2000). Furthermore, they usually require particular assumptions about the conditional distribution of the parameters and states. Alternatively, as proposed by Ansley and Kohn (1986), the PMSE of the estimated underlying states can be computed by using the Delta method and the first two moments of the asymptotic distribution of the parameter estimator. There are also corrections of the Kalman filter PMSE based on the Monte Carlo integration of the distribution of the parameter estimator which is approximated by the asymptotic distribution; see Hamilton (1986). However, the asymptotic distribution can be a poor approximation to the finite sample distribution when the sample size is not large enough. Proietti et al. (2007) implements the Hamilton (1986) and Ansley and Kohn (1986) procedures to obtain standard deviations of the Euro area output gap that incorporate the parameter uncertainty. However, Quenneville and Singh (2000) show that these two procedures miss terms of the same order as their proposed corrections. Consequently, they propose enhancements of both procedures to estimate the PMSE based on second order approximations of the parameter distribution and show that the corresponding reductions of the PMSE biases are relatively small. Kass and Steffey (1989) also propose a second order approximation which is even more computationally demanding. To overcome these limitations, Quenneville and Singh (2000) propose computing the PMSE of the underlying states by using the Monte Carlo integration of the distribution of the parameter estimator where this distribution is approximated by the posterior distribution obtained in a Bayesian fashion. Although their procedure reduces the biases in the PMSE, it is proposed in the context of a particular simple model, the local level model, and can be computationally demanding for more general unobserved component models. Finally, Pfeffermann and Tiller (2005) propose using bootstrap procedures to compute PMSE in the context of the Kalman filter. Bootstrap procedures have the advantage of being computationally simple even in relatively complicated models. Furthermore, they are robust against misspecification of the error distribution; see Wall and Stoffer $(2002,2004)$ and Rodríguez and Ruiz (2009) for their implementation to obtain the prediction distribution of future values of the observed variables. However, the bootstrap PMSE proposed by Pfeffermann and Tiller (2005) are designed to obtain unconditional PMSE of the estimates of the underlying states. The distinction between conditional and unconditional PMSE could be important in time-varying state space models with estimated parameters; see Ansley and Kohn (1986) for arguments in favor of computing conditional PMSE.

Consequently, in this paper, we propose two new bootstrap procedures to obtain conditional PMSE of the Kalman filter estimates of the unobserved states that incorporate the parameter uncertainty. Following, Hamilton (1986) and Quenneville and Singh (2000), the new procedures are based on the Monte Carlo integration of the distribution of the parameter estimator, but instead of approximating this distribution by the asymptotic or posterior distributions, we propose to approximate it by a bootstrap distribution; see Stoffer and Wall (1991) for the bootstrap approximation of the distribution of the Maximum Likelihood (ML) estimator of the parameters in state space models. The first procedure proposed in this paper is parametric in the sense that it is based on resampling from the assumed distribution of the errors. The second procedure is based on resampling from the residuals of the estimated model and consequently, it does not assume any particular error distribution. We carry out Monte Carlo experiments to analyze the finite sample performance of our procedures which is compared with that of alternative procedures. We show that the biases of the PMSE proposed in this paper are smaller than those of the asymptotic procedures of Hamilton (1986) and the bootstrap PMSE procedure of Pfeffermann and Tiller (2005). The results are illustrated with simulated and real data highlighting the importance of incorporating the parameter uncertainty in empirical applications.

The rest of the paper is organized as follows. Section 2 describes the Kalman filter and illustrates with simulated data the biases incurred when estimating the PMSE of the estimated underlying states by running the filter with estimated parameters. We also briefly describe the asymptotic procedure of Hamilton (1986) and the bootstrap procedures proposed by Pfeffermann and Tiller (2005) to overcome these biases. In Section 3, we propose two new bootstrap procedures to obtain PMSE of the one-step-ahead estimator of the unobserved states that take into account the parameter uncertainty. Their finite sample properties are analyzed and compared with those of the standard Kalman filter, the asymptotic and previously available bootstrap PMSE. Section 4 contains an empirical application in which we estimate the uncertainty associated with the unobserved quarterly output gap, Non-Accelerating Inflation Rate of Unemployment (NAIRU), investment rate and core inflation in the US. Finally, Section 5 concludes the paper.

\section{PMSE of Kalman filter estimates of states}

Consider the following state space model

$$
\begin{aligned}
& Y_{t}=Z_{t} \alpha_{t}+d_{t}+R_{1 t} \varepsilon_{t}, \\
& \alpha_{t}=T_{t} \alpha_{t-1}+c_{t}+R_{2 t} \eta_{t}, \quad t=1, \ldots, T,
\end{aligned}
$$

where $Y_{t}$ is an $N \times 1$ vector time series observed at time $t, \alpha_{t}$ is the $m \times 1$ vector of unobservable state variables, $\varepsilon_{t}$ is a $k \times 1$ vector of independent white noise processes with zero mean and covariance matrix $H_{t}$ and $\eta_{t}$ is a $g \times 1$ vector of serially uncorrelated disturbances with zero mean and covariance matrix $Q_{t}$. The disturbances $\varepsilon_{t}$ and $\eta_{t}$ are uncorrelated with each 
other in all time periods. Finally, the initial state vector, $\alpha_{1}$, has mean $a_{1 \mid 0}$ and covariance matrix $P_{1 \mid 0}$. All the system matrices, $Z_{t}, d_{t}, T_{t}, c_{t}, R_{1 t}, R_{2 t}, H_{t}$ and $Q_{t}$, are assumed to be known given the information available at time $t-1$. The model in (1) is time-invariant when, with the exception of $d_{t}$ and $c_{t}$, all the system matrices are time-invariant. For simplicity, in this paper, we focus on one-step-ahead estimates. However, the results can be easily extended to filtered and smoothed estimates of the states.

Next, we briefly describe the Kalman filter and previous proposals to obtain PMSE that incorporate the parameter uncertainty.

\subsection{PMSE of the Kalman filter}

The Kalman filter provides optimal unbiased linear one-step-ahead estimates of the underlying states, $\alpha_{t}$, and their corresponding PMSE, which are denoted by $a_{t \mid t-1}$ and $P_{t \mid t-1}$ respectively. If the errors are further assumed to have a conditional joint Normal distribution, then $a_{t \mid t-1}$ is the conditional mean of $\alpha_{t}$. In particular, the Kalman filter equations are given by

$$
\begin{aligned}
& a_{t \mid t-1}=T_{t} a_{t-1 \mid t-2}+c_{t}+K_{t} F_{t-1}^{-1} V_{t-1} \\
& P_{t \mid t-1}=T_{t} P_{t-1 \mid t-2} T_{t}^{\prime}-K_{t} F_{t-1}^{-1} K_{t}^{\prime}+R_{2 t} Q_{t} R_{2 t}^{\prime},
\end{aligned}
$$

where $K_{t}=T_{t} P_{t-1 \mid t-2} Z_{t-1}^{\prime}$ is the filter gain, $V_{t}=Y_{t}-d_{t}-Z_{t} a_{t \mid t-1}$ is the one-step-ahead vector of innovations and $F_{t}$ is their covariance matrix given by $F_{t}=Z_{t} P_{t \mid t-1} Z_{t}^{\prime}+R_{1 t} H_{t} R_{1 t}^{\prime}$; see, Harvey (1989) for details. It is important to observe that in linear models in which the system matrices are independent of the observations, the PMSE, $P_{t \mid t-1}$, is also independent of the observations. Therefore, in this case, $P_{t \mid t-1}$ is also the unconditional error covariance matrix associated with the conditional mean estimator of the underlying state.

For the implementation of the bootstrap procedures described later in this paper, it is also useful to express the state space model in (1) in what is known as the Innovation Form (IF) which depends on a unique disturbance vector instead of two. The IF is given by Eq. (2a) together with

$$
Y_{t}=Z_{t} a_{t \mid t-1}+d_{t}+V_{t} \text {. }
$$

Note that the unique disturbance vector in the IF is the one-step-ahead vector of innovations, $V_{t}$.

The implementation of the Kalman filter equations in (2) requires all the parameters involved in the system matrices and the initial conditions $a_{1 \mid 0}$ and $P_{1 \mid 0}$ being known. In practice, some of these parameters are unknown and, in order to run the filter, they are substituted by consistent estimates. In this paper, we consider the Quasi-Maximum Likelihood (QML) estimator of the parameters because of its good asymptotic and finite sample properties; see, for example, Harvey (1989) for details. Denote by $\widehat{Z}_{t}, \widehat{d}_{t}, \widehat{H}_{t}, \widehat{R}_{1 t}, \widehat{T}_{t}, \widehat{c}_{t}, R_{2 t}$ and $\widehat{Q}_{t}$ the system of matrices where the unknown parameters have been substituted by their QML estimates. Furthermore, the initial conditions for the filter are also unknown. The usual practice is to assume that they are given by the unconditional distribution of the unobserved states in case they are stationary or by a diffuse prior distribution when they are non-stationary; see Harvey (1989). Then, the Kalman filter can be run with the system matrices substituted by their respective estimates providing $\widehat{a}_{t \mid t-1}$ and $\widehat{P}_{t \mid t-1}$. Note that while $\widehat{a}_{t \mid t-1}$ is an estimate of the conditional mean of the state, $a_{t \mid t-1}, \widehat{P}_{t \mid t-1}$ is not the PMSE of $\widehat{a}_{t \mid t-1}$ as it does not take into account the parameter uncertainty involved in its computation. Therefore, $\widehat{P}_{t \mid t-1}$ will underestimate the true conditional PMSE of $\widehat{a}_{t \mid t-1}$ which is given by $\operatorname{PMSE}_{t \mid t-1}=E_{t-1}\left[\left(\widehat{a}_{t \mid t-1}-\alpha_{t}\right)\left(\widehat{a}_{t \mid t-1}-\alpha_{t}\right)^{\prime}\right]$ and can be decomposed as follows

$$
\operatorname{PMSE}_{t \mid t-1}=E_{t-1}\left[\left(a_{t \mid t-1}-\alpha_{t}\right)\left(a_{t \mid t-1}-\alpha_{t}\right)^{\prime}\right]+E_{t-1}\left[\left(\widehat{a}_{t \mid t-1}-a_{t \mid t-1}\right)\left(\widehat{a}_{t \mid t-1}-a_{t \mid t-1}\right)^{\prime}\right]
$$

where the $t-1$ under the expectation means that it is taken conditional on $\left\{Y_{1}, \ldots, Y_{t-1}\right\}$. Note that the cross-product $E_{t-1}\left[\left(\widehat{a}_{t \mid t-1}-a_{t \mid t-1}\right)\left(a_{t \mid t-1}-\alpha_{t}\right)^{\prime}\right]$ is zero under the assumption of conditional Normality. The first term in (4) measures filter uncertainty which is given by $P_{t \mid t-1}$. It represents how far would the state be from its estimate when the parameters are known. This uncertainty is due to the uncertainty in separating signal and noise and it is inherent to the Kalman filter. On the other hand, the second term in (4) measures the parameter uncertainty as it represents the discrepancy between the estimates of the unobserved states obtained with known and unknown parameters. $\widehat{P}_{t \mid t-1}$ measures the first uncertainty but fails to incorporate the second.

To illustrate the biases incurred when using $\widehat{P}_{t \mid t-1}$ to measure the true PMSE of $\widehat{a}_{t \mid t-1}$, we consider the following bivariate model proposed by Smets (2002) for the output gap

$$
\begin{aligned}
& \pi_{t}=\alpha(L) \pi_{t-1}+\beta z_{t-1}+\varepsilon_{t} \\
& y_{t}=y_{t}^{p}+z_{t} \\
& z_{t}=2 \theta_{1} \cos \theta_{2} z_{t-1}-\theta_{1}^{2} z_{t-2}+\lambda i_{t-1}+\eta_{t}^{z} \\
& y_{t}^{p}=\mu+y_{t-1}^{p}+\eta_{t}^{y}
\end{aligned}
$$




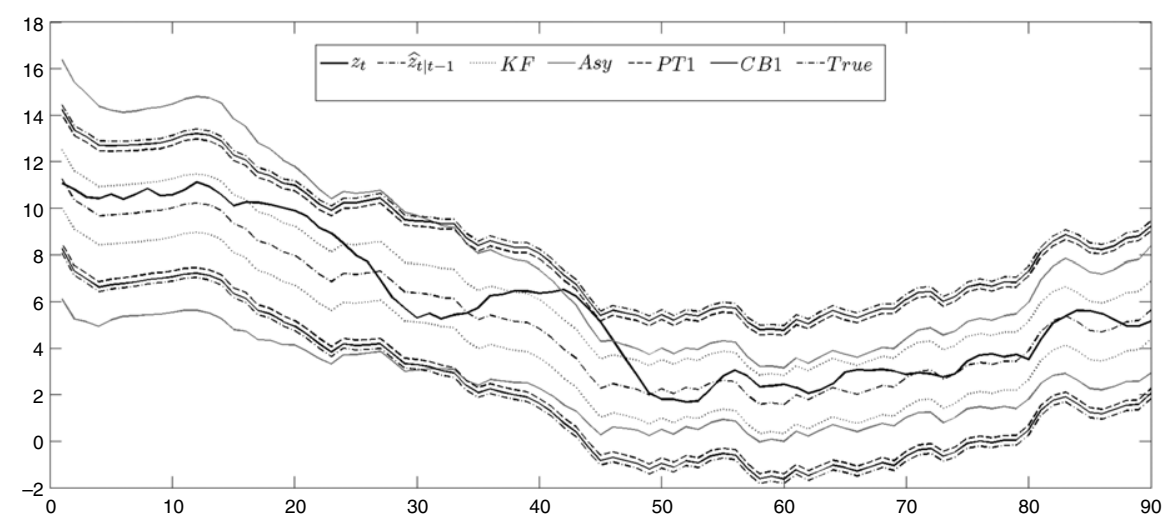

Fig. 1. Simulated and one-step-ahead estimates of the output gap together with $95 \%$ prediction intervals based on PMSE obtained from: (i) the Kalman filter with estimated parameters; (ii) the asymptotic approximation; (iii) the parametric bootstrap procedure of Pfeffermann and Tiller (2005); (iv) the conditional parametric bootstrap and $(v)$ the true conditional expression in Harvey and delle Monache (2009).

where the observed variables are the inflation, $\pi_{t}$, the product, $y_{t}$, and the exogenous real interest rate, $i_{t}$. The unobserved variables are the potential output, $y_{t}^{p}$, and the output gap, $z_{t}$. In order to simulate a time series from model (5), we have chosen the parameter values close to those estimated with real data by Smets (2002). In particular, in the Phillips-curve equation, $\alpha(L)$ is an order 4 polynomial with parameters $\alpha_{1}=0.4, \alpha_{2}=0.1, \alpha_{3}=0.2$ and $\alpha_{4}=0.1$ and $\beta=0.2$. The parameters of the reduced form aggregated demand equation are $\theta_{1}=0.77, \theta_{2}=0.24$ and $\lambda=-0.1$. Finally, all the disturbances in the model are mutually independent Gaussian white noises with variances $\sigma_{\varepsilon}^{2}=0.54, \sigma_{z}^{2}=0.05$ and $\sigma_{y}^{2}=1.2$. After generating a time series by model (5) with $T=100$, all the parameters are estimated by maximizing the Gaussian log-likelihood. ${ }^{1}$ Then, the Kalman filter is run with the estimated parameters. Fig. 1 plots the simulated output gap, $z_{t}$, and its one-step-ahead estimates, $\widehat{Z}_{t \mid t-1}$, together with their corresponding $95 \%$ intervals constructed using $\widehat{P}_{t \mid t-1}$. In order to avoid the effect of the initial values on the estimates of the Kalman filter PMSE, we have discarded the first ten $\widehat{P}_{t \mid t-1}$; see Ray (1989). The true PMSE of $\widehat{z}_{t \mid t-1}$ in (4) are computed using the formula in Harvey and delle Monache (2009) where the "true" and misspecified filters are the filters with known and estimated parameters respectively. The $95 \%$ prediction intervals constructed using the true PMSE are also plotted in Fig. 1 which shows that the intervals constructed using $\widehat{P}_{t \mid t-1}$ are much thinner than those based on the true PMSE. Furthermore, the true output gap, $z_{t}$, is too often out of the standard Kalman filter intervals which do not incorporate the parameter uncertainty. Therefore, these intervals can be misleading when used to measure the uncertainty around the estimated output gap.

The finite sample biases of $\widehat{P}_{t \mid t-1}$ as an estimator of the true PMSE in (4) have been obtained by the Monte Carlo simulation in the context of the following Random Walk plus Noise (RWN) model $^{2}$

$$
\begin{aligned}
& y_{t}=\mu_{t}+\varepsilon_{t} \\
& \mu_{t}=\mu_{t-1}+\eta_{t}
\end{aligned}
$$

where $y_{t}$ is the observation at time $t$ of the series of interest and $\eta_{t}$ is a Gaussian white noise with variance $\sigma_{\eta}^{2}=\sigma_{\varepsilon}^{2} q$ where $q=0.25$. We consider three alternative distributions for the measurement noise, $\varepsilon_{t}$, which is mutually independent of $\eta_{t}$. First, $\varepsilon_{t}$ is assumed to be a Gaussian white noise with variance $\sigma_{\varepsilon}^{2}=1$. Second, we assume that $\varepsilon_{t}$ is given by the following conditionally heteroscedastic process

$$
\varepsilon_{t}=\varepsilon_{t}^{\dagger} \sigma_{t},
$$

where $\sigma_{t}^{2}=\alpha_{0}+\alpha_{1} v_{t-1}^{2}$ with $\alpha_{0}=0.6719, \alpha_{1}=0.2, v_{t}$ is the innovation and $\varepsilon_{t}^{\dagger}$ is a Gaussian white noise process with variance 1 , distributed independently of $\eta_{t}$. The parameters in (7) have been chosen so that the marginal variance of $\varepsilon_{t}, \sigma_{\varepsilon}^{2}$, is 1 . Note that, given the specification of $\sigma_{t}^{2}$ and assuming that the parameters are known, the model is still conditionally Gaussian since knowledge of past observations implies knowledge of past innovations. ${ }^{3}$ Finally, we consider a non-conditionally Gaussian model by assuming that $\varepsilon_{t}$ is a serially independent sequence with a centered and

\footnotetext{
1 All programs for maximizing the log-likelihood and subsequent estimation of the unobserved components and PMSE were written by the first author in MATLAB.

2 Results for alternative univariate and multivariate state space models are available from the authors upon request. The conclusions are the same so we omit them to save space.

3 This model is related with the STARCH model described by Harvey et al. (1992) but they differ in that the STARCH model assumes that $\sigma_{t}^{2}=\alpha_{0}+\alpha_{1} \varepsilon_{t-1}^{2}$ and, consequently, it is not conditionally Gaussian.
} 
Table 1

Averages through time and Monte Carlo replicates of the percentage relative biases of the PMSE of the underlying level in the RWN model with Gaussian homoscedastic. Gaussian heteroscedastic and $\chi_{(1)}^{2}$ errors.

\begin{tabular}{lrrr}
\hline & Homoscedastic & Heteroscedastic & \multicolumn{1}{c}{$\chi_{(1)}^{2}$} \\
\hline & \multicolumn{3}{c}{$T=40$} \\
\hline KF & -13.56 & -16.49 & -16.28 \\
Asy & -6.94 & -7.76 & -7.16 \\
PT1 & -7.68 & -11.96 & -11.02 \\
PT2 & -12.06 & -15.96 & 2.49 \\
CB1 & -1.85 & -2.06 & -1.81 \\
CB2 & -2.92 & -2.08 & -3.82 \\
\hline & & $T=100$ & \\
& & -6.10 & -6.74 \\
KF & -6.50 & -2.36 & -4.21 \\
Asy & -3.12 & -3.99 & -4.44 \\
PT1 & -3.09 & -5.66 & 2.14 \\
PT2 & -4.89 & -0.92 & -1.74 \\
CB1 & -1.28 & -1.00 & -2.22 \\
CB2 & -1.27 & $T=500$ & \\
\hline & & -1.30 & -3.04 \\
& & -1.15 & -3.02 \\
KF & -0.99 & -0.93 & -2.64 \\
Asy & -0.96 & -0.98 & -1.84 \\
PT1 & -0.57 & -0.59 & -2.44 \\
PT2 & -0.64 & -0.59 & -2.51 \\
CB1 & -0.53 & & \\
CB2 & -0.45 & & \\
\hline & & &
\end{tabular}

re-scaled $\log \left(\chi_{(1)}^{2}\right)$ distribution. This distribution is of a particular interest in the context of Stochastic Volatility models; see, for instance, Harvey et al. (1994) for the relation between the RWN model in (6) and the linear transformation of the Autoregressive Stochastic Volatility model.

In each case, we generate $R=1000$ replicates of $\left\{y_{t}^{(j)}, t=1, \ldots, T ; j=1, \ldots, R\right\}$ by model (6) with sample sizes $T=40,100$ and 500. The initial values are chosen as $\mu_{0}=0$ and, when needed, $\sigma_{1}^{2}$ equal to the marginal variance of $\varepsilon_{t}$, which is one. For each replicate, the parameters are estimated by maximizing the Gaussian log-likelihood and the Kalman filter in (2) is run with estimated parameters and $\widehat{m}_{1 \mid 0}^{(j)}=0$ and $\widehat{P}_{1 \mid 0}^{(j)}=\infty$. The corresponding one-step-ahead estimates of the underlying level are denoted by $\widehat{m}_{t \mid t-1}^{(j)}$ and the Kalman filter PMSE are denoted by $\widehat{P}_{t \mid t-1}^{(j)}$. Furthermore, for each simulated series $j$, we compute the true PMSE of $\widehat{m}_{t \mid t-1}^{(j)}$ by the following expression derived from the results in Harvey and delle Monache (2009)

$$
\operatorname{PMSE}_{t \mid t-1}^{(j)}=\operatorname{PMSE}_{t-1 \mid t-2}^{(j)}\left(1-\widehat{K}_{t}^{(j)}\right)^{2}+\widehat{K}_{t}^{(j) 2} \sigma_{\varepsilon}^{2}+\sigma_{\eta}^{2}
$$

where $\widehat{K}_{t}^{(j)}$ is the filter gain obtained with estimated parameters. Table 1 reports the averages through the Monte Carlo replicates and time of the percentage relative biases given by $d_{t}^{(j)}=100 \cdot\left(\widehat{P}_{t \mid t-1}^{(j)} / \mathrm{PMSE}_{t \mid t-1}^{(j)}-1\right)$. In order to avoid the effect of the initial values on the estimates of the Kalman filter PMSE, we have discarded the first five $\widehat{P}_{t \mid t-1}^{(j)}$ in the averages reported in Table 1. Consider first the results when $\varepsilon_{t}$ is Gaussian. In this case, if $T=40, \widehat{P}_{t \mid t-1}$ underestimates by $13.56 \%$ the true PMSE of the one-step-ahead estimate of $\mu_{t}$, denoted by $\widehat{m}_{t \mid t-1}$; see Quenneville and Singh (2000) who report a bias of $-21.21 \%$ in a similar experiment. The difference between both results could be attributed to the fact that they compute smoothed estimates and, in order to avoid imposing positivity restrictions, they maximize the concentrated likelihood with respect to $\log \left(\sigma_{\eta}^{2}\right) / 2$. Table 1 also shows that the small sample biases of $\widehat{P}_{t \mid t-1}$ are slightly larger when the errors are conditionally heteroscedastic or non-Gaussian. In any case, note that given that the underestimation of the PMSE of $\widehat{m}_{t \mid t-1}$ can be attributed to the parameter uncertainty, it obviously decreases with the sample size.

The average biases reported in Table 1 show that when the Kalman filter is run with estimated parameters, $\widehat{P}_{t \mid t-1}$ is a negatively biased estimator of the conditional true PMSE of $\widehat{m}_{t \mid t-1}$. These biases can be rather large in small samples specially when the model is time-varying or non-Gaussian. Therefore, it is important to incorporate the parameter uncertainty when measuring the uncertainty associated with the estimates of the underlying states. As mentioned in the Introduction, there have been several procedures proposed in the literature with this goal. Next, we describe two of them. The first one is based on the Monte Carlo integration of the asymptotic distribution of the parameter estimator as proposed by Hamilton (1986). Second, we describe the bootstrap procedures proposed by Pfeffermann and Tiller (2005). 


\subsection{Asymptotic approximation}

Hamilton (1986) proposes to estimate the true PMSE of $\widehat{a}_{t \mid t-1}$ given in expression (4) by computing first the expectations conditional on the parameter estimates and then integrating over all possible values of the parameter estimates as follows

$$
\operatorname{PMSE}_{t \mid t-1}=E_{\widehat{\theta}}\left\{E_{t-1}\left[\left(a_{t \mid t-1}-\alpha_{t}\right)\left(a_{t \mid t-1}-\alpha_{t}\right)^{\prime} \mid \widehat{\theta}\right]\right\}+E_{\widehat{\theta}}\left\{E_{t-1}\left[\left(\widehat{a}_{t \mid t-1}-a_{t \mid t-1}\right)\left(\widehat{a}_{t \mid t-1}-a_{t \mid t-1}\right)^{\prime} \mid \widehat{\theta}\right]\right\},
$$

where $\widehat{\theta}$ is the estimated vector of model parameters. The expectations in (9) are obtained by integrating the distribution of the estimator of $\theta$ which is approximated by its asymptotic distribution. Therefore, Hamilton (1986) propose first to estimate the parameters and then obtain a large number, $M$, of realizations, $\widehat{\theta}_{a}^{(i)}$, from the asymptotic distribution of the estimator. The Kalman filter is run using each of the realizations $\widehat{\theta}_{a}^{(i)}$ and the original observations, $\left\{Y_{1}, \ldots, Y_{T}\right\}$, obtaining a series of estimates of the state and their corresponding PMSE, denoted by $\widehat{a}_{t \mid t-1}\left(\widehat{\theta}_{a}^{(i)}\right)$ and $\widehat{P}_{t \mid t-1}\left(\widehat{\theta}_{a}^{(i)}\right)$, respectively. ${ }^{4}$ An analogue of the first expectation within curly brackets in (9) can be obtained by $\widehat{P}_{t \mid t-1}\left(\widehat{\theta}_{a}^{(i)}\right)$ while the analogue of the second expectation is $\left[\widehat{a}_{t \mid t-1}\left(\widehat{\theta}_{a}^{(i)}\right)-\bar{a}_{t \mid t-1}\right]\left[\widehat{a}_{t \mid t-1}\left(\widehat{\theta}_{a}^{(i)}\right)-\bar{a}_{t \mid t-1}\right]^{\prime}$, where $\bar{a}_{t \mid t-1}=\frac{1}{M} \sum_{i=1}^{M} \widehat{a}_{t \mid t-1}\left(\widehat{\theta}_{a}^{(i)}\right)$. Then, after integrating over all values of $\widehat{\theta}$, the following approximation of the PMSE in (9) is obtained

$$
\widehat{\operatorname{PMSE}}_{t \mid t-1}^{\text {Asy }}=\frac{1}{M} \sum_{i=1}^{M} \widehat{P}_{t \mid t-1}\left(\widehat{\theta}_{a}^{(i)}\right)+\frac{1}{M} \sum_{i=1}^{M}\left[\widehat{a}_{t \mid t-1}\left(\widehat{\theta}_{a}^{(i)}\right)-\bar{a}_{t \mid t-1}\right]\left[\widehat{a}_{t \mid t-1}\left(\widehat{\theta}_{a}^{(i)}\right)-\bar{a}_{t \mid t-1}\right]^{\prime}
$$

To illustrate the performance of the asymptotic correction in (10), we consider again the same time series generated by the output gap model in (5). Fig. 1 plots the 95\% prediction intervals for $\widehat{z}_{t \mid t-1}$ obtained when the PMSE is computed as in (10). It is important to note that when extracting values from the asymptotic distribution of the Gaussian QML estimator of the parameters, it could be the case that the resulting extraction of some of the variances is non-positive. In these cases, we substitute the corresponding PMSE by the original $P_{t \mid t-1}$; see Quenneville and Singh (2000) for the same substitution. The 95\% prediction intervals plotted in Fig. 1 are wider than the Kalman filter intervals. However, note that when compared with the intervals constructed using the true PMSE, they are too wide at the beginning of the sample and too narrow at the end. They still leave out of the intervals some realizations of the true output gap.

The finite sample properties of the asymptotic correction in (10) have been analyzed in the context of the RWN model in (6). Table 1 reports the average relative biases of $\widehat{P M S E}_{t \mid t-1}^{\text {Asy }}$. We can observe that when $T=40, \widehat{\text { PMSE }}_{t \mid t-1}^{\text {Asy }}$ underestimates the true PMSE by $6.94 \%$ in the homoscedastic case and by a slightly larger percentage in the heteroscedastic and non-Gaussian models. Therefore, the bias in $\widehat{P}_{t \mid t-1}$ is reduced by approximately 50\%; compare with Quenneville and Singh (2000) who report a reduction of $15 \%$ for smoothed estimates of the underlying level based on a different estimator of the parameters. The underestimation observed in $\widehat{\mathrm{PMSE}}_{t \mid t-1}^{\text {Asy }}$ may be attributed to the inappropriateness in small samples of the asymptotic distribution to approximate the finite sample distribution of the QML estimator. Obviously, the QML estimator is consistent and the biases decrease with the sample size.

\subsection{Bootstrap procedures}

Bootstrapping has been seeing to have good properties to approximate the distribution of the ML estimator of the parameters of state space models and to obtain prediction densities of future values of the observed variables; see Stoffer and Wall (1991), Wall and Stoffer (2002, 2004) and Rodríguez and Ruiz (2009). Consequently, Pfeffermann and Tiller (2005) propose using bootstrap procedures to obtain the PMSE of $\widehat{a}_{t \mid t-1}$ that incorporate the parameter uncertainty. They propose parametric and non-parametric bootstrap procedures. The parametric procedure is based on extracting realizations, $\varepsilon_{t}^{*}$ and $\eta_{t}^{*}, t=1, \ldots, T$, from the joint true distribution of $\varepsilon_{t}$ and $\eta_{t}$, using them and the estimated parameters, $\widehat{\theta}$, substituted in model (1) to obtain a bootstrap replicate $\left\{Y_{1}^{*}, \ldots, Y_{T}^{*}\right\}$ as follows

$$
\begin{aligned}
Y_{t}^{*} & =\widehat{Z}_{t} \alpha_{t}^{*}+\widehat{d}_{t}+\widehat{R}_{1 t} \varepsilon_{t}^{*}, \\
\alpha_{t}^{*} & =\widehat{T}_{t} \alpha_{t-1}^{*}+\widehat{c}_{t}+\widehat{R}_{2 t} \eta_{t}^{*}, \quad t=1, \ldots, T .
\end{aligned}
$$

Note that this first step of the parametric bootstrap procedure is the same as the first step of the simulation smoother algorithm proposed by Durbin and Koopman (2002). Alternatively, the nonparametric bootstrap does not rely on any distributional assumption of the errors. Following Stoffer and Wall (1991), the standardized estimated innovations, $\widehat{V}_{t}^{s}=$ $\widehat{F}_{t}^{-1 / 2} \widehat{V}_{t}$, are resampled obtaining $\left\{V_{1}^{*}, \ldots, V_{T}^{*}\right\}$ and the bootstrap replicates, $\left\{Y_{1}^{*}, \ldots, Y_{T}^{*}\right\}$, are obtained from the IF in (3)

\footnotetext{
4 We add explicitly the dependence of the estimates of the unobserved states and their corresponding PMSE on the estimated parameters to clarify the procedure.
} 
and (2a) with the parameters substituted by their QML estimates and the innovations by their bootstrap counterparts as follows

$$
\begin{aligned}
& Y_{t}^{*}=\widehat{Z}_{t} a_{t \mid t-1}^{*}+\widehat{d}_{t}+V_{t}^{*} \\
& a_{t+1 \mid t}^{*}=\widehat{T}_{t+1} a_{t \mid t-1}^{*}+\widehat{c}_{t+1}+\widehat{K}_{t+1} \widehat{F}_{t}^{-1} V_{t}^{*} .
\end{aligned}
$$

Once a bootstrap replicate $\left\{Y_{1}^{*}, \ldots, Y_{T}^{*}\right\}$ has been obtained by any of the two alternative procedures in (11) and (12), the parameters are again estimated obtaining $\widehat{\theta}^{*}$.

When using the parametric resampling scheme, Pfeffermann and Tiller (2005) propose to compute the bootstrap PMSE as follows

$$
\widehat{\operatorname{PMSE}}_{t}^{\mathrm{PT} 1}=\widehat{P}_{t \mid t-1}-\frac{1}{B} \sum_{j=1}^{B} \widehat{P}_{t \mid t-1}\left(\widehat{\theta}^{*(j)}\right)+\frac{1}{B} \sum_{j=1}^{B}\left[\widehat{a}_{t \mid t-1}^{*}\left(\widehat{\theta}^{*(j)}\right)-\alpha_{t}^{*}\right]\left[\widehat{a}_{t \mid t-1}^{*}\left(\widehat{\theta}^{*(j)}\right)-\alpha_{t}^{*}\right]^{\prime}
$$

where $\widehat{P}_{t \mid t-1}\left(\widehat{\theta}^{*}\right)$ is obtained from the Kalman filter run with bootstrap estimates of the parameters, $\widehat{\theta}^{*}$, and the original series, $\left\{Y_{1}, \ldots, Y_{T}\right\}$. Then, the Kalman filter is run again using the bootstrap estimates of the parameters, $\widehat{\theta}^{*}$, and the bootstrap replicate of the series, $\left\{Y_{1}^{*}, \ldots, Y_{T}^{*}\right\}$, to obtain bootstrap estimates of the state, $\widehat{a}_{t \mid t-1}^{*}\left(\widehat{\theta}^{*}\right)$. Note that this estimator is unfeasible when implementing the non-parametric scheme as, in this case, $\alpha_{t}^{*}$ is not available. In this latter case, Pfeffermann and Tiller (2005) propose to obtain an alternative estimator of the PMSE which require a further run of the Kalman filter with the original estimates of the parameters, $\widehat{\theta}$, and the bootstrap replicate of the series, $\left\{Y_{1}^{*}, \ldots, Y_{T}^{*}\right\}$, to obtain estimates of the state denoted by $\widehat{a}_{t \mid t-1}^{*}(\widehat{\theta})$. After repeating the steps just described, $B$ times, the PMSE of $\widehat{a}_{t \mid t-1}$ proposed by Pfeffermann and Tiller (2005) is given by

$$
\widehat{\operatorname{PMSE}}_{t}^{\mathrm{PT} 2}=\frac{1}{B} \sum_{j=1}^{B}\left[\widehat{a}_{t \mid t-1}^{*}\left(\widehat{\theta}^{*(j)}\right)-\widehat{a}_{t \mid t-1}^{*(j)}(\widehat{\theta})\right]\left[\widehat{a}_{t \mid t-1}^{*}\left(\widehat{\theta}^{*(j)}\right)-\widehat{a}_{t \mid t-1}^{*(j)}(\widehat{\theta})\right]^{\prime}+2 \widehat{P}_{t \mid t-1}-\frac{1}{B} \sum_{j=1}^{B} \widehat{P}_{t \mid t-1}\left(\widehat{\theta}^{*(j)}\right) .
$$

In order to illustrate the performance of the parametric bootstrap PMSE in Eq. (13), we consider again the output gap model. Fig. 1, which plots the 95\% prediction intervals constructed using the parametric $\widehat{\mathrm{PMSE}}_{t}^{\mathrm{PT} 1}$, shows that the intervals are now much more close to the true $95 \%$ prediction intervals giving a more realistic picture about the uncertainty associated with the estimated output gap. The intervals based on the non-parametric PT2 are similar to those plotted in Fig. 1 for PT1.

The finite sample properties of the bootstrap PMSE in (13) and (14) have been analyzed using the same Monte Carlo design described above. Table 1, that reports the corresponding percentage biases, shows that, in the Gaussian homoscedastic model, the relative biases of the parametric $\widehat{\mathrm{PMSE}}_{t}^{\mathrm{PT} 1}$ are smaller than those of the Kalman filter with estimated parameters and similar to those of the asymptotic PMSE in (10). However, when $T=40$, the biases observed in the conditionally heteroscedastic and non-Gaussian models are much larger than when using the asymptotic approximation. When looking at the percentage biases of the nonparametric $\widehat{\mathrm{PMSE}}_{t}^{\mathrm{PT} 2}$, we can observed that they are hardly reduced in the two conditionally Gaussian models. The performance of the nonparametric bootstrap PMSE in the non-Gaussian model is rather surprising with the biases being positive. According to our simulation results, it seems that the non-parametric PMSE in (14) are not appropriate in small samples to estimate the uncertainty associated with the Kalman filter estimates of the unobserved states. Finally, note that, as expected, in large sample sizes, both procedures are approximately unbiased.

As described above, the PMSE in (13) and (14) are computed by taking expectations over all bootstrap realizations of the series and, consequently, it is not a conditional but an unconditional PMSE. However, the Kalman filter is designed to obtain conditional estimates of the underlying state and their corresponding PMSE. Therefore, it could be possible to improve the performance of bootstrap PMSE by computing them conditional on the available data set. This is the main idea behind the procedures proposed in this paper that we develop in the following section.

\section{New bootstrap procedures}

In this section, we propose two new bootstrap procedures to estimate the conditional PMSE of one-step-ahead estimates of the unobserved states obtained by implementing the Kalman filter with estimated parameters. Our proposed procedures are based on incorporating the parameter uncertainty by the Monte Carlo integration of the parameter estimator distribution. However, instead of dealing with the parameter uncertainty by simulating the parameters from the asymptotic distribution, as in Hamilton (1986), or by using the Bayes posterior distribution as in Quenneville and Singh (2000), we simulate them from a bootstrap distribution. Stoffer and Wall (1991) show that, in small sample sizes, the bootstrap distribution is a better approximation to the finite sample distribution of the ML estimator than the asymptotic approximation. Consequently, the bootstrap PMSE proposed in this paper are expected to have better properties in small samples than those of Hamilton (1986). On the other hand, our procedure is computationally simpler than that proposed by Quenneville and Singh (2000) and can be easily implemented in relatively complicated models. Finally, the new bootstrap procedures have two advantages when compared with the bootstrap procedures proposed by Pfeffermann and Tiller (2005). 
First, for each bootstrap replicate, the Kalman filter has to be run once instead of three times as in the non-parametric bootstrap in (14) or twice as in the parametric bootstrap in (13). Consequently, the procedure is computationally simpler. Furthermore, by computing the conditional PMSE, the finite sample biases are smaller.

We also propose resampling using parametric and nonparametric schemes. As in Pfeffermann and Tiller (2005), the parametric bootstrap procedure is based on resampling from the assumed joint true distribution of the noises as in (11). Alternatively, the nonparametric procedure is based on resampling from the empirical distribution of the standardized onestep-ahead innovations, $\widehat{V}_{t}^{s}$, and obtain bootstrap replicates of the series as in (12). Once a bootstrap replicate $\left\{Y_{1}^{*}, \ldots, Y_{T}^{*}\right\}$ is obtained by any of the two resampling schemes, the parameters are estimated by QML, obtaining $\widehat{\theta}^{*}$. Then, the Kalman filter is run once with the original observations $\left\{Y_{1}, \ldots, Y_{T}\right\}$ and $\widehat{\theta}^{*}$ to obtain $\widehat{a}_{t \mid t-1}\left(\widehat{\theta}^{*}\right)$ and $\widehat{P}_{t \mid t-1}\left(\widehat{\theta}^{*}\right), t=1, \ldots, T$. After obtaining $B$ bootstrap replicates, the conditional bootstrap PMSE is obtained as follows

$$
\widehat{\operatorname{PMSE}}_{t \mid t-1}^{\mathrm{CB}}=\frac{1}{B} \sum_{j=1}^{B} \widehat{P}_{t \mid t-1}\left(\widehat{\theta}^{*(j)}\right)+\frac{1}{B} \sum_{j=1}^{B}\left[\widehat{a}_{t \mid t-1}\left(\widehat{\theta}^{*(j)}\right)-\bar{a}_{t \mid t-1}^{*}\right]\left[\widehat{a}_{t \mid t-1}\left(\widehat{\theta}^{*(j)}\right)-\bar{a}_{t \mid t-1}^{*}\right]^{\prime}
$$

where $\bar{a}_{t \mid t-1}^{*}=\frac{1}{B} \sum_{j=1}^{B} \widehat{a}_{t \mid t-1}\left(\widehat{\theta}^{*(j)}\right)$. We denote by $\widehat{\mathrm{PMSE}}_{t \mid t-1}^{\mathrm{CB} 1}$ the PMSE obtained using the parametric scheme while $\widehat{\mathrm{PMSE}}_{t \mid t-1}^{\mathrm{CB} 2}$ corresponds to the nonparametric bootstrap. Note that in the new procedures proposed in this paper, the Kalman filter is run with the bootstrap estimates of the parameters and the original time series. In this way, the PMSE in Eq. (15) is conditional on the information contained in the original series. Furthermore, by computing the conditional PMSE, we avoid running the filter two more times as in the procedure proposed by Pfeffermann and Tiller (2005). Consequently, our procedure is computationally simpler.

In order to illustrate the performance of the bootstrap PMSE proposed in this paper, we consider again the output gap model. Fig. 1 plots the 95\% intervals obtained using the parametric $\widehat{\mathrm{PMSE}}_{t \mid t-1}^{\mathrm{CB} 1}$ in (15). In this particular example, the prediction intervals are very close to those obtained when using the $\widehat{\text { PMSE }}^{\text {PT1 }}$ although they are slightly closer to the true intervals.

Finally, the finite sample properties of the two new bootstrap PMSE are analyzed by simulation using the same Monte Carlo designs described above. Table 1, that reports the relative biases, shows that, regardless of the model, the biases of the proposed parametric and non-parametric conditional bootstrap PMSE are very similar between them and clearly smaller than those observed when the PMSE are computed using the Kalman filter with estimated parameters, the asymptotic approximation of Hamilton (1986) or the bootstrap procedures of Pfeffermann and Tiller (2005). The averages reported in Table 1 show that the reductions of the relative biases can be very important when $T=40$. For example, in the homoscedastic RWN model, the relative bias of the nonparametric bootstrap is reduced by a $79 \%$ with respect to the bias of the PMSE computed by the Kalman filter with estimated parameters. The reduction is $58 \%$ with respect to the asymptotic approximation and $62 \%$ and $76 \%$ with respect to the parametric and nonparametric bootstrap procedures of Pfeffermann and Tiller (2005) respectively. The reduction of the relative biases is still important when $T=100$ while when $T=500$ all PMSE are approximately unbiased. It is also remarkable that the relative biases of the parametric and non-parametric bootstrap procedures proposed in this paper are approximately the same. The good performance of the parametric procedure could be expected given that we are resampling from the true error distribution. However, it is comforting to observe that the behavior of the non-parametric procedure, which does not assume any particular distribution, is comparable with that of the parametric procedure. Given that the biases of the parametric and nonparametric resampling schemes are so similar, it seems safer to always use the nonparametric procedure which does not rely on any particular assumption on the error distribution.

Therefore, our simulation results show that in small and moderate sample sizes, the conditional PMSE of $\widehat{a}_{t \mid t-1}$ computed using the new bootstrap procedures proposed in this paper have very small biases giving a realistic measure of the uncertainty associated with the underlying estimated states. Furthermore, this reduction of bias is accomplished using procedures which are simple from a computational point of view and, consequently, they can be easily implemented in practice.

\section{Empirical application: estimating the output gap, the NAIRU, the trend investment rate and the core inflation in US}

In this section, we apply the new proposed bootstrap PMSE of the estimated unobserved states to estimate the uncertainty associated with the estimation of the output gap, NAIRU, investment trend and core inflation of the US economy, based on the unobserved component model proposed by Doménech and Gómez (2006). The logarithm of the GDP, $y_{t}$, the inflation rate defined as the average inflation over the last four months, $\pi_{t}$, the unemployment rate also defined as the average of unemployment rate over the last four months, $U_{t}$ and the nominal investment rate defined as the ratio between investment and GDP, $x_{t}$ have been observed quarterly from 1948:Q1 to 2003:Q1.

The model proposed by Doménech and Gómez (2006) incorporates the following three stylized facts often observed in those macroeconomic variables, namely: (i) Negative correlation between the output gap and the deviations of unemployment from the NAIRU, often known as the Okun law, (ii) Short run trade-off between inflation and unemployment known as 
forward looking Phillips curve, and (iii) Co-movement of output and investment called accelerator-type investment equation. The model is given by

$$
\begin{aligned}
& y_{t} \equiv y_{t}^{p}+z_{t}, \\
& z_{t+1}=2 \theta_{1} \cos \theta_{2} z_{t}-\theta_{1}^{2} z_{t-1}+\omega_{z t}, \\
& y_{t+1}^{p}=\bar{\mu}+y_{t}^{p}+\omega_{y t}, \\
& \pi_{t}=\left(1-\sum_{i=1}^{4} \mu_{i}\right) \bar{\pi}_{t}+\sum_{i=1}^{4} \mu_{i} \pi_{t-i}+\eta_{y} z_{t}+v_{\pi t}, \\
& \bar{\pi}_{t}=\bar{\pi}_{t-1}+\omega_{\pi t}, \\
& U_{t}=\phi_{u} U_{t-1}+\left(1-\phi_{u}\right) \bar{U}_{t}+\phi_{0} z_{t}+v_{u t}, \\
& \bar{U}_{t}=\bar{U}_{t-1}+\omega_{u t}, \\
& x_{t}=\beta_{x} x_{t-1}+\left(1-\beta_{x}\right) \bar{x}_{t}+\beta_{y_{0}} z_{t}+\beta_{y_{1}} z_{t-1}+v_{x t}, \\
& \bar{x}_{t}=\bar{x}_{t-1}+\omega_{x t},
\end{aligned}
$$

where $z_{t}$ is the unobserved output gap which is assumed to follow a cyclical AR(2) process in Eq. (16b) and, $y_{t}^{p}$ is the logarithm of the potential output represented by a random walk plus drift model in Eq. (16c). The parameter $\bar{\mu}$ captures the growth rate of the potential output. The noises $\omega_{z t}$ and $\omega_{y t}$ are assumed to be mutually independent white noise processes with zero mean and variances $\sigma_{\omega z}^{2}$ and $\sigma_{\omega y}^{2}$ respectively. The following two equations, (16d) and (16e) describe the dynamic evolution of inflation, $\pi_{t}$ and its relation with the output gap. $\bar{\pi}_{t}$ is the core inflation which follows a random walk. The noises $v_{\pi t}$ and $\omega_{\pi t}$ are white noise processes with variances $\sigma_{v \pi}^{2}$ and $\sigma_{\omega \pi}^{2}$ respectively. Both noises are mutually independent and independent of $\omega_{z t}$ and $\omega_{y t}$. Eqs. (16f) and (16g) describe the Okun law where $\bar{U}_{t}$ is the NAIRU. Once more, the disturbances associated with the unemployment, $v_{u t}$ and $\omega_{u t}$ are white noises with variances $\sigma_{v u}^{2}$ and $\sigma_{\omega u}^{2}$, respectively. They are mutually independent and independent of the rest of disturbances in the model. Finally, the last two equations, (16h) and (16i) describe the dynamic evolution of the investment rate, $x_{t}$, where $\bar{x}_{t}$ is the long run investment trend. The disturbances $v_{x t}$ and $\omega_{x t}$ are white noise processes with zero mean and variances $\sigma_{v x}^{2}$ and $\sigma_{\omega x}^{2}$ and, once more, they are assumed to be mutually independent and independent of all previous disturbances.

Model (16) can be casted into a state space framework as in (1) with $Y_{t}=\left[y_{t}, U_{t}-\phi_{u} U_{t-1}, x_{t}-\beta_{x} x_{t-1}, \pi_{t}-\right.$ $\left.\left(\sum_{i=1}^{4} \mu_{i} \pi_{t-i}\right)\right]^{\prime}, \alpha_{t}=\left[y_{t}^{p}, \bar{U}_{t}, \bar{x}_{t}, \bar{\pi}_{t}, z_{t-2}, z_{t-1}, z_{t}\right]^{\prime}, \varepsilon_{t}=\left[v_{u t}, v_{x t}, v_{\pi t}\right], \eta_{t}=\left[\omega_{y t}, \omega_{u t}, \omega_{x t}, \omega_{\pi t}, \omega_{z t}\right], H_{t}=\operatorname{diag}\left\{\sigma_{v u}^{2}\right.$, $\left.\sigma_{v x}^{2}, \sigma_{v \pi}^{2}\right\}, Q_{t}=\operatorname{diag}\left\{\sigma_{\omega y}^{2}, \sigma_{\omega u}^{2}, \sigma_{\omega x}^{2}, \sigma_{\omega \pi}^{2}, \sigma_{\omega z}^{2}\right\}$,

$$
\begin{aligned}
T & =\left[\begin{array}{ccccccc}
1 & 0 & 0 & 0 & 0 & 0 & 0 \\
0 & 1 & 0 & 0 & 0 & 0 & 0 \\
0 & 0 & 1 & 0 & 0 & 0 & 0 \\
0 & 0 & 0 & 1 & 0 & 0 & 0 \\
0 & 0 & 0 & 0 & 0 & 1 & 0 \\
0 & 0 & 0 & 0 & 0 & 0 & 1 \\
0 & 0 & 0 & 0 & 0 & -\theta_{1}^{2} & 2 \theta_{1} \cos \theta_{2}
\end{array}\right], \\
Z & =\left[\begin{array}{ccccccc}
1 & 0 & 0 & 0 & 0 & 0 & 0 \\
0 & 1-\phi_{u} & 0 & 0 & 0 & 0 & \phi_{0} \\
0 & 0 & 1-\beta_{x} & 0 & 0 & \beta_{y_{1}} & \beta_{y_{0}} \\
0 & 0 & 0 & 1-\sum_{i=1}^{4} \mu_{i} & 0 & 0 & \eta_{y}
\end{array}\right] \\
R_{1} & =\left[\begin{array}{ccccc}
1 & 0 & 0 & 0 & 0 \\
0 & 1 & 0 & 0 & 0 \\
0 & 0 & 1 & 0 & 0 \\
0 & 0 & 0 & 1 & 0 \\
0 & 0 & 0 & 0 & 0 \\
0 & 0 & 0 & 0 & 0 \\
0 & 0 & 0 & 0 & 1
\end{array}\right], \text { and } R_{2}=\left[\begin{array}{ccc}
0 & 0 & 0 \\
1 & 0 & 0 \\
0 & 1 & 0 \\
0 & 0 & 1
\end{array}\right]
\end{aligned}
$$

where diag $\{$.$\} is the diagonal matrix.$

After a preliminary analysis of the data, several breaks in the marginal variances of inflation and output are detected and incorporated into de model; see Doménech and Gómez (2006). Table 2 reports the QML estimates of the parameters together with their standard asymptotic deviations obtained numerically using the results in Harvey (1989) for the asymptotic distribution of the QML estimator of the hyperparameters of state-space models. With respect to the estimates reported 

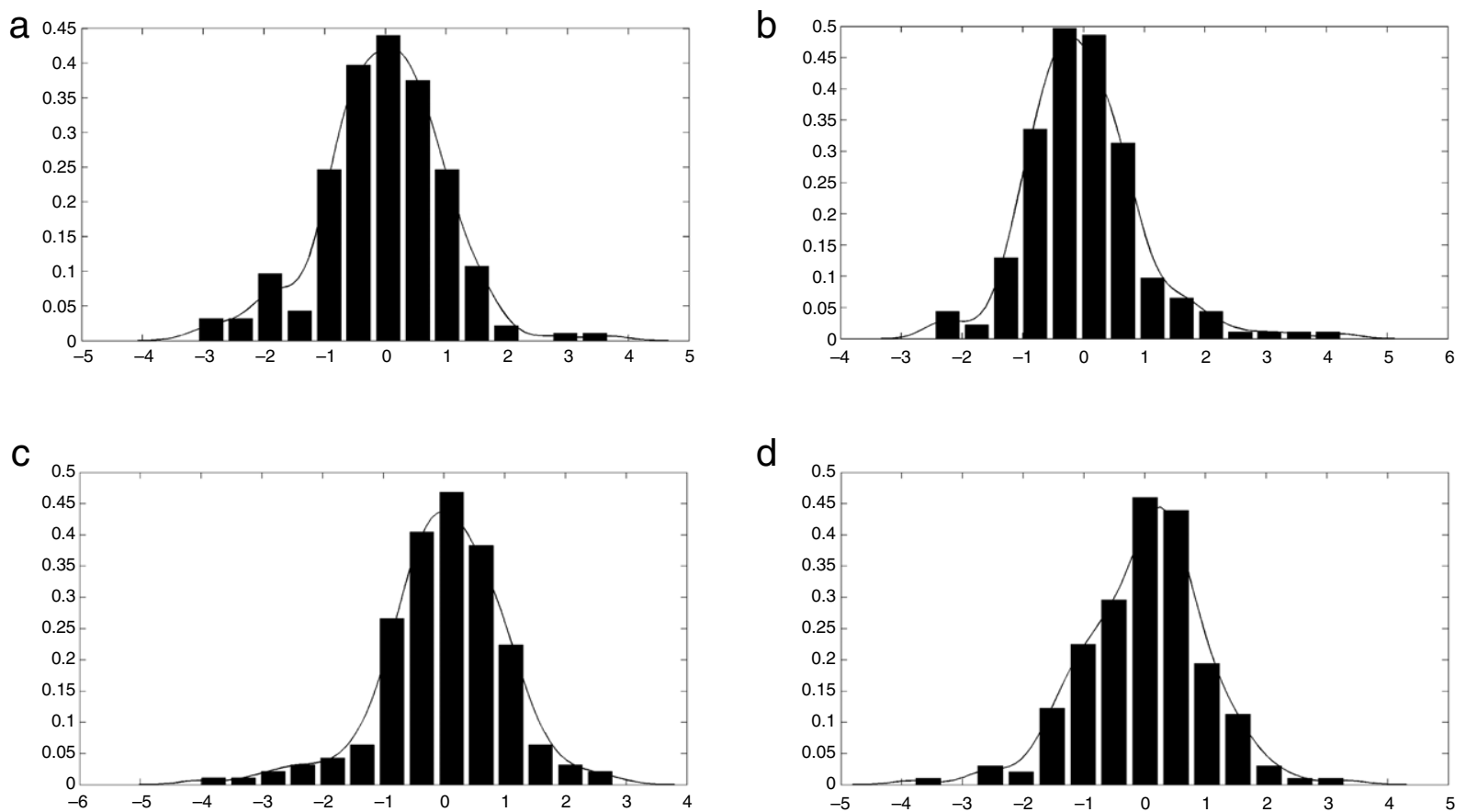

Fig. 2. Histogram and kernel estimates of the density of standardized one-step-ahead innovations of (a) Output, (b) Unemployment, (c) Investment and (d) Inflation.

Table 2

QML parameter estimates of multivariate unobserved component model for output, unemployment, inflation and investment. Estimation based on 221 quarterly observations from 1948:Q1 to 2003:Q1. Standard deviations in parenthesis.

\begin{tabular}{|c|c|c|c|c|c|c|c|c|c|}
\hline \multicolumn{2}{|l|}{ Output } & \multicolumn{4}{|c|}{ Inflation } & \multicolumn{2}{|c|}{ NAIRU } & \multicolumn{2}{|c|}{ Investment } \\
\hline$\theta_{1}$ & $\begin{array}{l}0.7710 \\
(0.0490)\end{array}$ & $\eta_{y}$ & $\begin{array}{l}0.3123 \\
(0.0887)\end{array}$ & $\sigma_{v \pi}$ & $\begin{array}{l}0.0044 \\
(0.0015)\end{array}$ & $\phi_{0}$ & $\begin{array}{l}-0.3107 \\
(0.0217)\end{array}$ & $\beta_{y_{0}}$ & $\begin{array}{l}0.6458 \\
(0.0397)\end{array}$ \\
\hline$\theta_{2}$ & $\begin{array}{l}0.2359 \\
(0.0781)\end{array}$ & $\mu_{1}$ & $\begin{array}{l}0.1427 \\
(0.0637)\end{array}$ & $\sigma_{\omega \pi_{1}}$ & $\begin{array}{l}0.0096 \\
(0.0011)\end{array}$ & $\phi_{U}$ & $\begin{array}{l}0.3653 \\
(0.0582)\end{array}$ & $\beta_{y_{1}}$ & $\begin{array}{l}-0.6102 \\
(0.0473)\end{array}$ \\
\hline$\sigma_{\omega y_{1}}$ & $\begin{array}{l}0.0075 \\
(0.0007)\end{array}$ & $\mu_{2}$ & $\begin{array}{l}-0.1332 \\
(0.0498)\end{array}$ & $\sigma_{\omega \pi_{2}}$ & $\begin{array}{l}0.0163 \\
(0.0037)\end{array}$ & $\sigma_{v u}$ & $\begin{array}{l}0.0009 \\
(0.0002)\end{array}$ & $\beta_{x}$ & $\begin{array}{l}0.8253 \\
(0.0548)\end{array}$ \\
\hline$\sigma_{\omega y_{2}}$ & $\begin{array}{l}0.0069 \\
(0.0008)\end{array}$ & $\mu_{3}$ & $\begin{array}{l}0.2413 \\
(0.0771)\end{array}$ & $\sigma_{\omega \pi_{3}}$ & $\begin{array}{l}0.0047 \\
(0.0013)\end{array}$ & $\sigma_{\omega u}$ & $\begin{array}{l}0.0022 \\
(0.0003)\end{array}$ & $\sigma_{v u}$ & $\begin{array}{l}0.0033 \\
(0.0003)\end{array}$ \\
\hline$\sigma_{\omega z}$ & $\begin{array}{l}0.0048 \\
(0.0003)\end{array}$ & $\mu_{4}$ & $\begin{array}{l}-0.1679 \\
(0.0714)\end{array}$ & & & & & $\sigma_{\omega u}$ & $\begin{array}{l}0.0023 \\
(0.0007)\end{array}$ \\
\hline
\end{tabular}

in Table 2, which are very close to those reported by Doménech and Gómez (2006), it is interesting to point out that the estimated break in the output volatility is highly significant with a decrease in the volatility after 1983:Q1. Moreover, the inflation volatility has a substantial increase in 1972:Q1 and a decrease in 1983:Q1. The sign of the coefficients associated with the output gap are significant and positive in the inflation, unemployment and investment equations.

Fig. 2, which plots kernel estimates of each of the densities of the four components of the innovation vector, shows that the unemployment and investment innovations seem to have asymmetric distributions. Therefore, it could be expected that the parametric based on the Normal assumption and nonparametric bootstrap PMSE may differ in these cases.

The Kalman filter is run with the QML estimates of the parameters reported in Table 2 to obtain one-step-ahead estimates of the underlying components and their PMSE. We also estimate the PMSE by using the asymptotic approximation, the parametric and nonparametric bootstrap procedures of Pfeffermann and Tiller (2005) and the two new conditional bootstrap procedures proposed in this paper. The parametric resampling schemes are based on assuming Gaussian innovations. Table 3 reports the averages and standard deviations through time of the PMSE estimated for each of the four underlying components by each of the five procedures. First of all, note that the PMSE computed using the non-parametric bootstrap procedure of Pfeffermann and Tiller (2005) are even smaller than when they are computed using the Kalman filter with estimated parameters. As shown in the simulation results reported in previous section, this procedure seems to be not appropriated to measure the uncertainty associated with the unobserved components. Alternatively, the PMSE obtained by the Kalman filter with estimated parameters and by the asymptotic procedure are very similar for the NAIRU, investment and long-run inflation. However, there is a large difference in the PMSE of the output gap which is 0.0143 when estimated by the Kalman filter and 0.0214 when incorporating the parameter uncertainty using the asymptotic approximation. Furthermore, 
Table 3

Averages and standard deviations (in parenthesis) through time of PMSE computed using the Kalman filter with estimated parameters (KF), the asymptotic approximation (Asy), the parametric (PT1) and non-parametric (PT2) bootstrap procedures of Pfeffermann and Tiller (2005) and the parametric (CB1) and non-parametric (CB2) new bootstrap procedures.

\begin{tabular}{lllllll}
\hline & KF & Asy & PT1 & PT2 & CB1 & 0.0238 \\
Output gap & 0.0143 & 0.0214 & 0.0217 & 0.0104 & 0.0283 \\
& $(0.0012)$ & $(0.0013)$ & $(0.0016)$ & $(0.0082)$ & $(0.0078)$ & 0.0063 \\
NAIRU & 0.0050 & 0.0051 & 0.0051 & 0.0026 & $(0.0021)$ \\
Investment & $(0.0004)$ & $(0.0004)$ & $(0.0004)$ & $(0.0023)$ & 0.0089 \\
& 0.0059 & 0.0062 & 0.0093 & 0.0031 & $(0.0074)$ \\
Inflation & $(0.0019)$ & $(0.0021)$ & $(0.0048)$ & $(0.0043)$ & $(0.0066)$ & 0.0223 \\
& 0.0137 & 0.0140 & 0.0142 & $(0.0097)$ \\
\hline
\end{tabular}
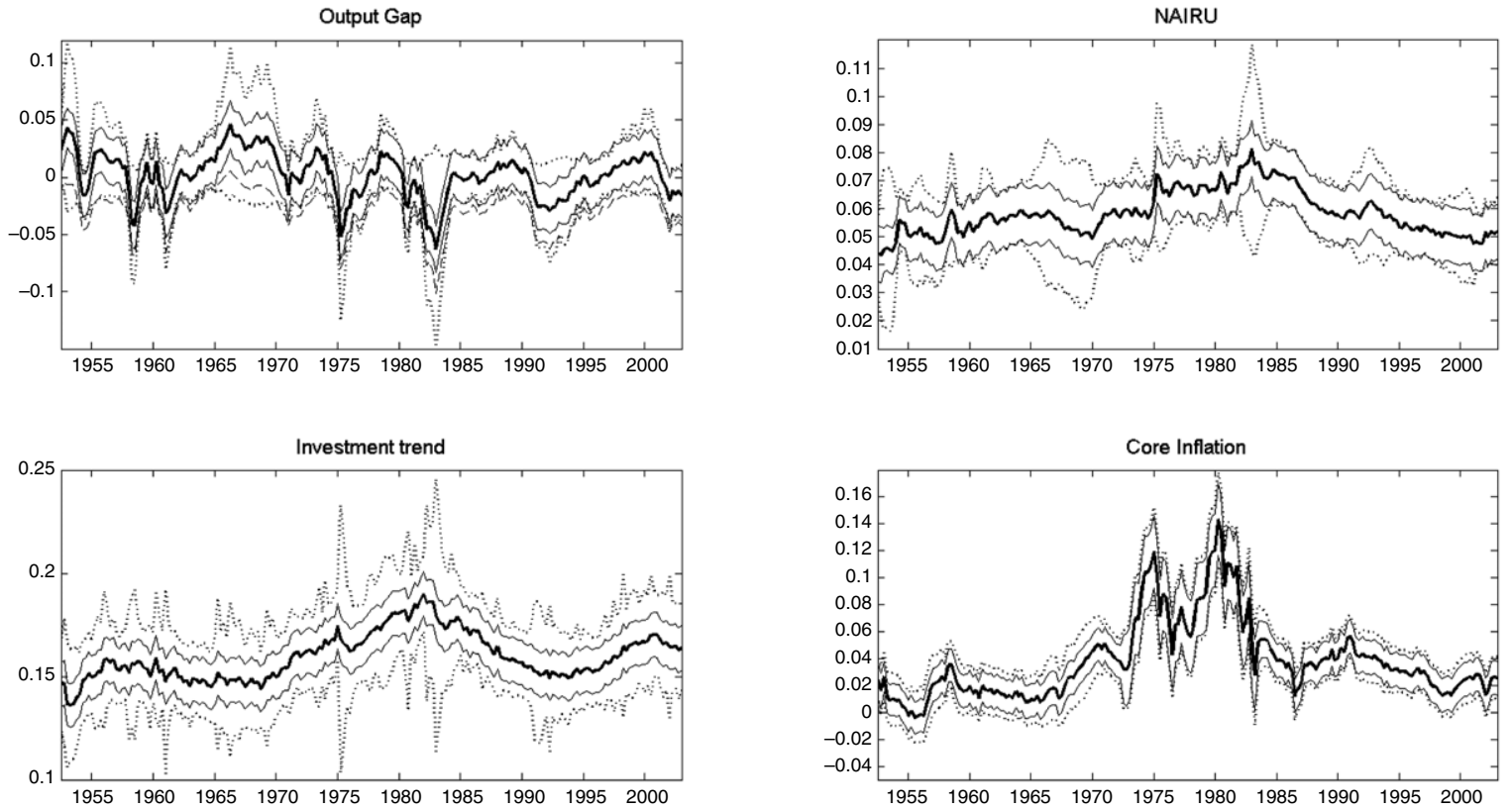

Fig. 3. One-step-ahead Kalman filter estimates of the output gap, NAIRU, Investment trend and Core inflation together with the corresponding $90 \%$ intervals based on PMSE constructed using: (i) the Kalman filter with estimated parameters (continuous lines) and (ii) the non-parametric conditional bootstrap (dotted lines).

in concordance with our Monte Carlo results, the PMSE estimated using the parametric bootstrap procedure proposed by Pfeffermann and Tiller (2005) are very similar to those obtained by using the asymptotic procedure for all variables but investment. In this case, the PMSE computed using the bootstrap procedure is 0.0093 , while it is estimated as 0.0062 using the asymptotic approximation. Finally, the PMSE obtained using the two bootstrap procedures proposed in this paper are clearly larger than those obtained by all the alternative procedures for all four unobserved variables. Note that the parametric bootstrap is based on the assumption of Gaussian errors which seems to be not satisfied in all equations. As expected, given the simulation results in the previous section, the PMSE estimated using the parametric and non-parametric bootstrap procedures are very similar for all variables. Only in the case of the NAIRU, the PMSE estimated using the parametric bootstrap is 0.0063 while it is 0.0089 when the non-parametric procedure is implemented. Note that the unemployment is one of the variables for which the innovations seem to be non-Gaussian. Also note that the bootstrap PMSE for the investment trend is around five times the PMSE computed using the Kalman filter. The smallest difference between the bootstrap and the Kalman filter PMSE is about 30\% for the core inflation. Consequently, the prediction intervals based on the PMSE proposed by Hamilton (1986) and Pfeffermann and Tiller (2005) will be wider than those based on the PMSE of the Kalman filter. Furthermore, when the bootstrap PMSE proposed in this paper are used for constructing prediction intervals, the resulting intervals will be even wider than for the previous procedures. This is reflected in Fig. 3 that plots one-step-ahead estimates of the output gap, NAIRU, investment trend and the core inflation together with their $90 \%$ prediction intervals based on the PMSE estimated by the Kalman filter and by the nonparametric bootstrap procedure proposed in this paper. It is clear that the latter intervals are much wider than the former. Therefore, taking into account the parameter uncertainty may change the conclusions about the uncertainty associated to the four unobserved variables estimated. This effect is specially important when estimating the NAIRU and the long-run investment rate. The differences between the prediction intervals constructed 


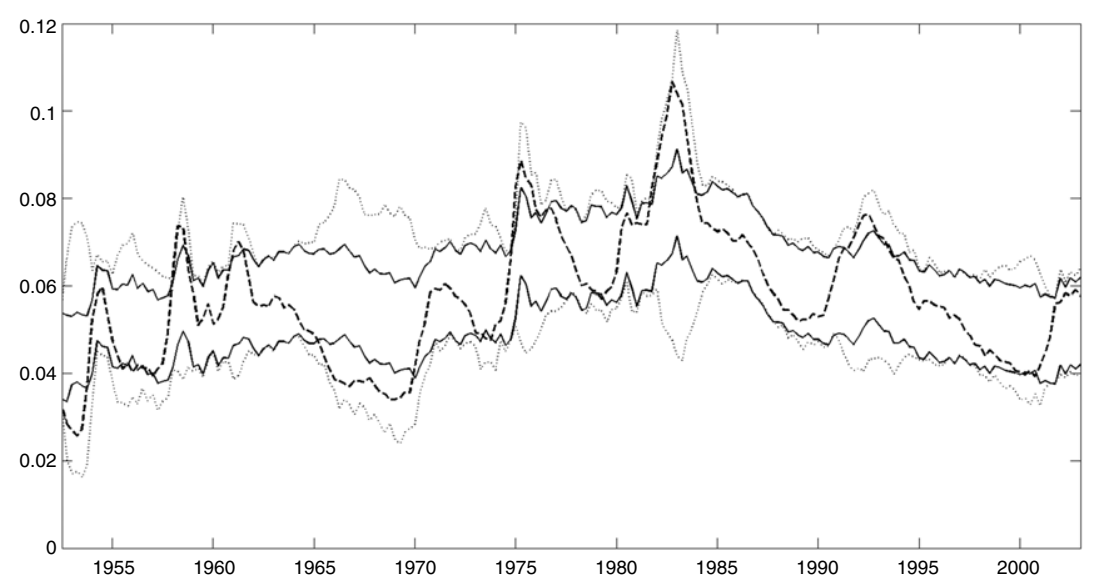

Fig. 4. Observed unemployment rate together with the corresponding $90 \%$ intervals of the NAIRU based on PMSE constructed using: (i) the Kalman filter with estimated parameters (continuous lines) and (ii) the non-parametric conditional bootstrap (dotted lines).

for the NAIRU may have important implications as regards to its utility for macroeconomic policy. Fig. 4, that plots the unemployment rate together with the 90\% prediction intervals for the NAIRU, shows that the former is out of the intervals in the second half of the sixties indicating an expansion and in the first half of the eighties suggesting a recession. Given this result, Doménech and Gómez (2006) conclude that the difference between the NAIRU and the unemployment rate is useful for policy makers in the sense that it can be used for identifying expansions and recessions very accurately. However, once we construct the intervals by taking into account the parameter uncertainty, as suggested in this paper, they are much wider and, consequently, the unemployment is not out of the $90 \%$ prediction intervals of the NAIRU at any moment along the sample period considered. ${ }^{5}$ Therefore, when taking into account the parameter uncertainty, the conclusion of Staiger et al. (2001) that doubt about the ability of that difference for economy policy is supported.

\section{Conclusions}

In this paper, we propose two new bootstrap procedures to obtain PMSE of the Kalman filter estimator of the unobserved states in state space models which take into account the uncertainty attributable to parameter estimation. These procedures have the advantage of being computationally simple having at the same time good finite sample properties. In the context of the RWN model, we show that their biases are smaller than those of the PMSE computed using the Kalman filter, the asymptotic approximation and alternative bootstrap procedures previously proposed in the literature.

We illustrate, using simulated and real data, the importance of taking into account the parameter uncertainty. In the context of US macroeconomic data, we put some doubts on the usefulness of the difference between the unemployment rate and the NAIRU for predicting expansions and recessions of the economy once the parameter uncertainty is taken into account.

\section{Acknowledgment}

The second author gratefully acknowledges the financial support from Project ECO2009-08100 by the Spanish Government. The usual disclaimers apply.

\section{References}

Ansley, C.F., Kohn, R., 1986. Prediction mean squared error for state space models with estimated parameters. Biometrika 73, 467-473.

Carter, C.K., Kohn, R., 1994. On Gibbs sampling for state space models. Biometrika 81 (3), 541-553.

Doménech, R., Gómez, V., 2006. Estimating potential output, core inflation, and the NAIRU as latent variables. Journal of Business and Economic Statistics $24,354-365$.

Durbin, J., Koopman, S., 2000. Time series analysis of non-Gaussian observations based on state space models from both classical and Bayesian perspectives. With comments. Journal of the Royal Statistical Society Series B 62, 3-56.

Durbin, J., Koopman, S., 2002. A simple and efficient simulation smoother for state space time series analysis. Biometrika 89, 603-615.

Fernández-Villaverde, J., Rubio-Ramírez, J.F., Sargent, T.J., Watson, M.W., 2007. ABCs (and Ds) of understanding VARs. The American Economic Review 97 (3), 1021-1026.

Hamilton, J.D., 1986. A standard error for the estimated state vector of a state-space model. Journal of Econometrics 33, 387-397.

\footnotetext{
5 Doménech and Gómez (2006) implement a smoothing algorithm to estimate the unobserved components instead of the one-step-ahead estimates considered in this paper. However, due to the large correlations between smoothed and predicted estimates, their estimates are comparable with those obtained in this paper.
} 
Harvey, A., 1989. Forecasting, Structural Time Series Models and the Kalman Filter. Cambridge University Press, Cambridge.

Harvey, A., 2000. Comments of "time series analysis of non-Gaussian observations based on state space models from both classical and Bayesian perspectives". Journal of the Royal Statistical Society Series B 62, 34-35.

Harvey, A., delle Monache, D., 2009. Computing the mean square error of unobserved components extracted by misspecified time series models. Journal of Economic Dynamics \& Control 33, 283-295.

Harvey, A., Ruiz, E., Sentana, E., 1992. Unobserved component time series models with ARCH disturbances. Journal of Econometrics 52, $129-157$.

Harvey, A., Ruiz, E., Shephard, N.G., 1994. Multivariate stochastic variance models. The Review of Economic Studies 61, $247-264$.

Kass, R.E., Steffey, D., 1989. State space models with a common stochastic variance. Journal of the American Statistical Association 84, 717-726.

Orphanides, A., van Norden, S., 2002. The unreliability of output-gap estimates in real time. The Review of Economics and Statistics 84, 569-583.

Pfeffermann, D., Tiller, R., 2005. Bootstrap approximation to prediction MSE for state-space models with estimated parameters. Journal of Time Series Analysis 26, 893-916.

Proietti, T., Musso, A., Westermann, T., 2007. Estimating potential output and the output gap for the euro area: a model-based production function approach. Empirical Economics 33, 85-113.

Quenneville, B., Singh, A.C., 2000. Bayesian prediction mean squared error for state space models with estimated parameters. Journal of Time Series Analysis 21, 219-236.

Ray, W.D., 1989. Rates of convergence to steady state for the linear growth version of a dynamic linear model (DLM). International Journal of Forecasting 5, 537-545

Rodríguez, A., Ruiz, E., 2009. Bootstrap prediction intervals in state-space models. Journal of Time Series Analysis 30, 167-178.

Smets, F., 2002. Output gap uncertainty: does it matter for the Taylor rule? Empirical Economics 27, 113-129.

Staiger, D., Stock, J.H. Watson, M.W., 2001. Prices, Wages and the US NAIRU in the 1990s. National Bureau of Economic Research, WP8320.

Stock, J.H., Watson, M.W., 2007. Why has US inflation become harder to forecast? Journal of Money, Credit and Banking 39, 3-33.

Stoffer, D.S., Wall, K.D., 1991. Bootstrap state-space models: Gaussian maximum likelihood and the Kalman filter. Journal of the American Statistical Association 86, 1024-1033.

Wall, K.D. Stoffer, D.S., 2002. A state space approach to bootstrapping conditional forecasts in ARMA models. Journal of Time Series Analysis 23, 733-751.

Wall, K.D., Stoffer, D.S., 2004. Resampling in state space models, In: Harvey, A.C., Koopman, S.J., Shephard, N.G. (Eds.), State Space and Unobserved Component Models: Theory and Applications. Cambridge University Press, Cambridge. 